\title{
A GALERKIN METHOD FOR THE FORWARD-BACKWARD HEAT EQUATION
}

\author{
A. K. AZIZ AND J.-L. LIU
}

\begin{abstract}
In this paper a new variational method is proposed for the numerical approximation of the solution of the forward-backward heat equation. The approach consists of first reducing the second-order problem to an equivalent first-order system, and then using a finite element procedure with continuous elements in both space and time for the numerical approximation. Under suitable regularity assumptions, error estimates and the results of some numerical experiments are presented.
\end{abstract}

\section{INTRODUCTION}

In this paper we consider a new Galerkin method for approximating the following parabolic boundary value problem:

$$
\begin{gathered}
\sigma(x, t) \phi_{t}(x, t)-\phi_{x x}(x, t)=f(x, t) \quad \forall(x, t) \in \Omega, \\
\begin{cases}\phi( \pm 1, t)=0 & \forall t \in[0,1], \\
\phi(x, 0)=0 & \forall x \in[0,1] \\
\phi(x, 1)=0 & \forall x \in[-1,0],\end{cases}
\end{gathered}
$$

where $\Omega=(-1,1) \times(0,1)$, and the coefficient $\sigma(x, t)$ changes sign in $\Omega$.

Problems of the type $\sigma \phi_{t}=\phi_{x x}$ with $\sigma$ taking both positive and negative values appear to have been considered by Gevrey in [5, 6], who specifically treated the case $\sigma(x, t)=x^{m}$ with $m$ an odd integer. Much later, in 1968, a detailed treatment of the case $\sigma(x, t)=x$ was given by Baouendi and Grisvard [3]. A similar treatment in a context where the second-order derivative is replaced by a suitable nonlinear differential operator may be found in Lions' book [10]. Recently, Goldstein and Mazumdar proved [7] that problem (1.1), (1.2) is well posed in a suitable function space.

Problem (1.1), (1.2) arises in boundary layer problems in fluid dynamics (cf. Stewartson $[11,12]$ and the references contained therein), in plasma physics, and in astrophysics in the study of propagation of an electron beam through the solar corona (see LaRosa [8]).

Received August 7, 1989; revised January 19, 1990 and March 1, 1990.

1980 Mathematics Subject Classification (1985 Revision). Primary 65N30, 35K20.

Key words and phrases. Finite element, first-order system, error estimates. 
As far as the numerical treatment of $(1.1),(1.2)$ is concerned, very little can be found in the literature. In [14] this problem is dealt with by a finite difference method, where a rather delicate piecing together on the dividing line is considered. The main drawback of this approach is that it requires a highdegree regularity of solutions in order to obtain a reasonable rate of convergence. For example, in [14] it is required that the solution possesses continuous derivatives of order 4 in $x$ and order 2 in $t$ to obtain the rate of convergence $\mathbf{O}\left(k+h^{2}\right)$, where $k$ and $h$ are mesh sizes in time and in space, respectively. These regularity assumptions appear to be unrealistic in view of the fact that the solution may not even be $H^{1}$ in $t$.

By a change of dependent variables,

$$
\mathbf{u}=\left[\begin{array}{l}
u_{1} \\
u_{2}
\end{array}\right], \quad u_{1}=e^{-\lambda t} \phi, u_{2}=e^{-\lambda t} \phi_{x}
$$

equation (1.1) may be written as the symmetric first-order system

$$
A_{1} \mathbf{u}_{x}+A_{2} \mathbf{u}_{t}+A_{3} \mathbf{u}=\mathbf{f}
$$

where

$$
A_{1}=\left[\begin{array}{cc}
0 & -1 \\
-1 & 0
\end{array}\right], \quad A_{2}=\left[\begin{array}{ll}
\sigma & 0 \\
0 & 0
\end{array}\right], \quad A_{3}=\left[\begin{array}{cc}
\lambda \sigma & 0 \\
0 & 1
\end{array}\right], \quad \mathbf{f}=\left[\begin{array}{c}
e^{-\lambda t} f \\
0
\end{array}\right]
$$

We shall examine a finite element procedure for the numerical approximation of the solution for this system of first-order partial differential equations. Our results show that the $L^{2}$ rate of convergence is $\mathbf{O}\left(h^{k}\right)$, where $h$ is the mesh size of space and time, if the solution $\mathbf{u} \in\left(H^{k+1}(\Omega)\right)^{2}$.

The finite element approximation for first-order systems in connection with the mixed type equations has been studied by Aziz, Leventhal, and Werschulz [2]. Many finite element methods for the heat equation have been proposed and analyzed in the literature (cf. Thomée [13]). A common approach, often referred to as the method of lines, is to first apply the Galerkin method in space to reduce the heat equation to a set of ordinary differential equations. Then a suitable method is applied to integrate the ordinary differential equation. However, our problem (1.1), (1.2) does not fit into this category, simply because the coefficient $\sigma(x, t)$ changes sign, i.e., $\sigma(x, t) \geq 0$ for $x \geq 0$ and $\sigma(x, t)<0$ for $x<0$. In contrast to the method of lines described above, we use finite elements to discretize the first-order system (1.3) in space and time simultaneously.

The use of continuous finite element methods to discretize time-dependent problems has been proposed in the past. For example, Aziz and Monk [1] proposed a continuous finite element method for the second-order heat equation; however, it does not appear that this method can be extended to our problem. Lesaint and Raviart [9] also proposed a collocation method for solving the heat 
equation, rewritten as a first-order positive symmetric system; however, our first-order system is not positive in the sense of Friedrichs [4].

\section{NotATION AND DEFINITIONS}

Let $\Omega$ be a bounded domain in the $(x, t)$ plane with boundary $\partial \Omega$. We denote by $\mathbf{n}=\left(n_{x}, n_{t}\right)$ the outward unit vector normal to $\partial \Omega$.

We consider the following problem: Given a vector-valued function $\mathbf{f}=$ $\left(f_{1}, f_{2}\right) \in\left(L^{2}(\Omega)\right)^{2}$, find a vector-valued function $\mathbf{u}=\left(u_{1}, u_{2}\right): \Omega \rightarrow \mathbf{R}^{2}$, which is a solution of the first-order system

$$
L \mathbf{u} \equiv A_{1} \mathbf{u}_{x}+A_{2} \mathbf{u}_{t}+A_{3} \mathbf{u}=\mathbf{f} \text { in } \Omega,
$$

with the boundary condition

$$
M \mathbf{u} \equiv u_{1}=0 \quad \text { on } \Gamma,
$$

where $\Gamma \equiv \Gamma_{2} \cup \Gamma_{3} \cup \Gamma_{5} \cup \Gamma_{6}$ and the $\Gamma_{i}$ are defined as follows:

$$
\begin{aligned}
& \Gamma_{1}=\{(x, t): x \in[-1,0], t=0\}, \\
& \Gamma_{2}=\{(x, t): x=-1, t \in[0,1]\}, \\
& \Gamma_{3}=\{(x, t): x \in[-1,0], t=1\}, \\
& \Gamma_{4}=\{(x, t): x \in[0,1], t=1\}, \\
& \Gamma_{5}=\{(x, t): x=1, t \in[0,1]\}, \\
& \Gamma_{6}=\{(x, t): x \in[0,1], t=0\},
\end{aligned}
$$

hence $\partial \Omega=\Gamma_{1} \cup \cdots \cup \Gamma_{6}$. In order to give a weak formulation of problem (2.1), (2.2), we define a $2 \times 2$ matrix-valued function $T$ and a function space $V$ as follows:

$$
T \mathbf{v}=\left[\begin{array}{cc}
\alpha & 0 \\
\beta \sigma & \alpha
\end{array}\right] \mathbf{v},
$$

where $\alpha$ and $\beta$ are known functions in $x$ and $t$ to be specified such that $T$ is bounded, and

$$
V=\left\{\mathbf{u} \in\left(H^{1}(\Omega)\right)^{2}: M \mathbf{u}=0\right\} .
$$

We shall make constant use of the classical Sobolev space $H^{m}(\Omega)$ provided with the norm

and the seminorm

$$
\|v\|_{m, \Omega}=\left(\sum_{|\alpha| \leq m} \int_{\Omega}\left|\partial^{\alpha} v\right|^{2} d x\right)^{1 / 2}
$$

where $\alpha$ is a multi-index.

$$
|v|_{m, \Omega}=\left(\sum_{|\alpha|=m} \int_{\Omega}\left|\partial^{\alpha} v\right|^{2} d x\right)^{1 / 2},
$$

Define the bilinear form $B: V \times V \rightarrow \mathbf{R}$ by $B(\mathbf{u}, \mathbf{v})=(L \mathbf{u}, T \mathbf{v})$, where $(\cdot, \cdot)$ denotes the $\left(L^{2}(\Omega)\right)^{2}$ inner product. Thus the weak formulation of $(2.1)$ for a given $\mathbf{f} \in\left(L^{2}(\Omega)\right)^{2}$ is: To find a $\mathbf{u} \in V$ such that

$$
B(\mathbf{u}, \mathbf{v})=(\mathbf{f}, T \mathbf{v}) \quad \forall \mathbf{v} \in V .
$$




\section{The Galerkin Procedure}

In this section we shall derive an a priori estimate for the solution of $(2.3)$ and describe our finite element scheme.

We assume the constants $k_{1}>0$ and $k_{2}>0$ are chosen so that

$$
\begin{aligned}
& H 1: \lambda \alpha \sigma-\frac{1}{2}(\alpha \sigma)_{t}+\frac{1}{2}(\beta \sigma)_{x} \geq k_{1}, \\
& H 2: \alpha \geq k_{2}, \\
& H 3: \alpha_{x}+\beta \sigma<2 \sqrt{k_{1} k_{2}}, \\
& H 4:\left.\sigma n_{t}\right|_{\Gamma_{1} \cup \Gamma_{4}} \geq 0 .
\end{aligned}
$$

Now we state the fundamental result of this section as

Theorem 3.1. If $H 1-H 4$ hold, then there exists a constant $C$ depending only on the constants $k_{1}$ and $k_{2}$ such that

$$
\|\mathbf{u}\|_{0, \Omega}^{2} \leq C B(\mathbf{u}, \mathbf{u}) \quad \forall \mathbf{u} \in V .
$$

Proof. We have

$$
\begin{aligned}
B(\mathbf{u}, \mathbf{u})=(L \mathbf{u}, T \mathbf{u})=\int_{\Omega}\left(\sigma \alpha u_{1_{1}} u_{1}-\alpha u_{2_{x}} u_{1}\right. & +\lambda \sigma \alpha u_{1}^{2}-\beta \sigma u_{1} u_{1_{x}} \\
& \left.-\alpha u_{1_{x}} u_{2}+\beta \sigma u_{1} u_{2}+\alpha u_{2}^{2}\right) d \Omega .
\end{aligned}
$$

Since

$$
\begin{aligned}
\sigma \alpha u_{1_{t}} u_{1} & =\frac{1}{2}\left(\sigma \alpha u_{1}^{2}\right)_{t}-\frac{1}{2}(\sigma \alpha)_{t} u_{1}^{2} \\
-\alpha u_{2_{x}} u_{1}-\alpha u_{1_{x}} u_{2} & =-\left(\alpha u_{1} u_{2}\right)_{x}+\alpha_{x} u_{1} u_{2}, \\
-\beta \sigma u_{1} u_{1_{x}} & =-\frac{1}{2}\left(\beta \sigma u_{1}^{2}\right)_{x}+\frac{1}{2}(\beta \sigma)_{x} u_{1}^{2}
\end{aligned}
$$

we now let

$$
\begin{aligned}
& I_{1}=\int_{\Omega}\left[\left(-\frac{1}{2}(\sigma \alpha)_{t}+\lambda \alpha \sigma+\frac{1}{2}(\beta \sigma)_{x}\right) u_{1}^{2}+\left(\alpha_{x}+\beta \sigma\right) u_{1} u_{2}+\alpha u_{2}^{2}\right] d \Omega, \\
& I_{2}=\int_{\Omega}\left[\left(\frac{1}{2} \alpha \sigma u_{1}^{2}\right)_{t}-\left(\alpha u_{1} u_{2}+\frac{1}{2} \beta \sigma u_{1}^{2}\right)_{x}\right] d \Omega .
\end{aligned}
$$

Applying Green's formula to $I_{2}$, we obtain

$$
I_{2}=\int_{\Gamma_{1} \cup \cdots \cup \Gamma_{6}}\left(\frac{1}{2} \alpha \sigma n_{t} u_{1}^{2}-\alpha n_{x} u_{1} u_{2}-\frac{1}{2} \beta \sigma n_{x} u_{1}^{2}\right) d s .
$$

From the boundary conditions we then have:

$$
\begin{aligned}
& \text { on } \Gamma_{2} \cup \Gamma_{3} \cup \Gamma_{5} \cup \Gamma_{6}: I_{2}=0, \text { since } u_{1}=0 ; \\
& \text { on } \Gamma_{1}: n_{x}=0, n_{t}=-1, \text { and by } H 2 \text { and } H 4, I_{2} \geq 0 ; \\
& \text { on } \Gamma_{4}: n_{x}=0, n_{t}=1, \text { and by } H 2 \text { and } H 4, I_{2} \geq 0 .
\end{aligned}
$$

By $H 1$ and $H 2$, we have

$$
I_{1} \geq \int_{\Omega}\left[k_{1} u_{1}^{2}-\left(\alpha_{x}+\beta \sigma\right)\left|u_{1}\right|\left|u_{2}\right|+k_{2} u_{2}^{2}\right] d \Omega .
$$


If $H 3$ holds, then it is possible to choose $0<c_{1}<k_{1}$ and $0<c_{2}<k_{2}$ such that $\alpha_{x}+\beta \sigma<2 \sqrt{c_{1} c_{2}}<2 \sqrt{k_{1} k_{2}}$. Since $-2 \sqrt{c_{1} c_{2}}\left|u_{1}\right|\left|u_{2}\right| \geq-c_{1} u_{1}^{2}-c_{2} u_{2}^{2}$, we get

$$
I_{1} \geq \int_{\Omega}\left[\left(k_{1}-c_{1}\right) u_{1}^{2}+\left(k_{2}-c_{2}\right) u_{2}^{2}\right] d \Omega .
$$

The result now follows with $1 / C=\min \left\{k_{1}-c_{1}, k_{2}-c_{2}\right\}$.

To approximate problem (2.3), we in essence replace the Hilbert space $V$ by a finite-dimensional subspace $V^{h}$ which satisfies the boundary condition (2.2). Here, $h>0$ is a real parameter such that as $h \rightarrow 0, \operatorname{dim} V^{h} \rightarrow \infty$. The Galerkin approximation is: Find a $\mathbf{u}^{h} \in V^{h}$ such that

$$
B\left(\mathbf{u}^{h}, \mathbf{v}^{h}\right)=\left(\mathbf{f}, T \mathbf{v}^{h}\right) \quad \forall \mathbf{v}^{h} \in V^{h} .
$$

Equation (3.2) is equivalent to a set of linear equations. Indeed, let $\left\{\phi^{j}\right\}_{j=1}^{n}$ be a basis for $V^{h}$ and denote

$$
\mathbf{u}^{h}=\left[\begin{array}{l}
u_{1}^{h} \\
u_{2}^{h}
\end{array}\right], \quad \phi^{j}=\left[\begin{array}{l}
\phi_{1}^{j} \\
\phi_{2}^{j}
\end{array}\right], \quad N^{j}=\left[\begin{array}{cc}
\phi_{1}^{j} & 0 \\
0 & \phi_{2}^{j}
\end{array}\right]
$$

then

$$
\begin{aligned}
\mathbf{u}^{h} & =\left[\begin{array}{l}
u_{1}^{h} \\
u_{2}^{h}
\end{array}\right]=\left[\begin{array}{ll}
\sum_{j=1}^{n} u_{1}^{j} \phi_{1}^{j} \\
\sum_{j=1}^{n} & u_{2}^{j} \phi_{2}^{j}
\end{array}\right] \\
& =\sum_{j=1}^{n}\left[\begin{array}{cc}
\phi_{1}^{j} & 0 \\
0 & \phi_{2}^{j}
\end{array}\right]\left[\begin{array}{l}
u_{1}^{j} \\
u_{2}^{j}
\end{array}\right]=\sum_{j=1}^{n} N^{j} \mathbf{u}^{j},
\end{aligned}
$$

where

$$
\mathbf{u}^{j}=\left[\begin{array}{l}
u_{1}^{j} \\
u_{2}^{j}
\end{array}\right] .
$$

If we denote $U=\left(\mathbf{u}^{1}, \ldots, \mathbf{u}^{n}\right)^{T}$ and $\mathbf{b}=\left(\mathbf{b}^{1}, \ldots, \mathbf{b}^{n}\right)^{T}$ with

$$
\mathbf{b}^{j}=\left[\begin{array}{l}
b_{1}^{j} \\
b_{2}^{j}
\end{array}\right]=\left(\mathbf{f}, T N^{j}\right), \quad 1 \leq j \leq n,
$$

then $U$ is given by the linear system

$$
\mathbf{A} U=\mathbf{b},
$$

where $\mathbf{A}=\left(a_{i j}\right)_{1 \leq i, j \leq n}$ and $a_{i j}=\left(L N^{j}, T N^{i}\right)$.

Lemma 3.1. A is invertible.

Proof. Suppose that there is a vector $Z$ such that $\mathbf{A} Z=0$. Letting

$$
\mathbf{z}^{h}=\sum_{j=1}^{n} N^{j} \mathbf{z}^{j}
$$


we find that

$$
\begin{aligned}
Z^{T} \mathbf{A} Z & =\left(\mathbf{z}^{1^{T}}, \ldots, \mathbf{z}^{n^{T}}\right) \mathbf{A}\left[\begin{array}{c}
\mathbf{z}^{1} \\
\vdots \\
\mathbf{z}^{n}
\end{array}\right]=\sum_{i=1}^{n} \mathbf{z}^{i^{T}} \mathbf{A}_{i} Z \\
& =\sum_{i=1}^{n} \mathbf{z}^{i^{T}}\left(\sum_{j=1}^{n}\left(L N^{j}, T N^{i}\right) \mathbf{z}^{j}\right)=\sum_{i=1}^{n} \mathbf{z}^{i^{T}}\left(L \mathbf{z}^{h}, T N^{i}\right) \\
& =\left(L \mathbf{z}^{h}, T \mathbf{z}^{h}\right)=B\left(\mathbf{z}^{h}, \mathbf{z}^{h}\right) ;
\end{aligned}
$$

since $Z^{T} \mathbf{A} Z=0$, and by (3.1), we then have

$$
C\left\|\mathbf{z}^{h}\right\|_{0, \Omega}^{2} \leq B\left(\mathbf{z}^{h}, \mathbf{z}^{h}\right)=0 .
$$

Hence $\mathbf{z}^{h}=0$. Now $\left\{\phi^{j}\right\}_{j=1}^{n}$ is linearly independent (being a basis for $V^{h}$ ), so that (3.4) and $\mathbf{z}^{h}=0$ imply that $Z=0$. Since $\mathbf{A}$ is a square matrix with trivial nullspace, $\mathbf{A}$ is invertible.

We now prove existence, uniqueness, and uniform stability of solutions to (3.2).

Theorem 3.2. If $H 1-H 4$ hold, then there is a unique $\mathbf{u}^{h} \in V^{h}$ satisfying (3.2). Moreover, there exists a constant $C$ depending only on the constants $k_{1}$ and $k_{2}$ such that

$$
\left\|\mathbf{u}^{h}\right\|_{0, \Omega} \leq C\|\mathbf{f}\|_{0, \Omega}
$$

Proof. The existence and uniqueness follow from Lemma 3.1; inequality (3.5) is an immediate consequence of Theorem 3.1 and the boundedness of $T$.

\section{ERROR ANALYSIS}

In this section we shall derive $L^{2}$ error estimates for the Galerkin approximation problem (3.2). The problem of estimating the error may be reduced to a problem in approximation theory.

Theorem 4.1. Let $\mathbf{u}$ and $\mathbf{u}^{h}$ be solutions of problems (2.3) and (3.2), respectively. If $\mathrm{H1-H4}$ hold, then there exists a $\mathrm{C}>0$ depending only on the constants $k_{1}$ and $k_{2}$ such that

$$
\left\|\mathbf{u}-\mathbf{u}^{h}\right\|_{0, \Omega} \leq C \inf _{\mathbf{v}^{h} \in V^{h}}\left\|\mathbf{u}-\mathbf{v}^{h}\right\|_{1, \Omega} .
$$

Proof. Given $\mathbf{v}^{h} \in V^{h}$, we use Theorem 3.1 to find

$$
\begin{aligned}
C_{1}\left\|\mathbf{u}^{h}-\mathbf{v}^{h}\right\|_{0, \Omega}^{2} & \leq B\left(\mathbf{u}^{h}-\mathbf{v}^{h}, \mathbf{u}^{h}-\mathbf{v}^{h}\right) \\
& =\left(L\left(\mathbf{u}-\mathbf{v}^{h}\right), T\left(\mathbf{u}^{h}-\mathbf{v}^{h}\right)\right) \leq C_{2}\left\|\mathbf{u}-\mathbf{v}^{h}\right\|_{1, \Omega}\left\|\mathbf{u}^{h}-\mathbf{v}^{h}\right\|_{0, \Omega} .
\end{aligned}
$$

Setting $C_{3}=C_{2} / C_{1}$, we find

$$
\left\|\mathbf{u}^{h}-\mathbf{v}^{h}\right\|_{0, \Omega} \leq C_{3}\left\|\mathbf{u}-\mathbf{v}^{h}\right\|_{1, \Omega} .
$$


Since

$$
\left\|\mathbf{u}-\mathbf{u}^{h}\right\|_{0, \Omega} \leq\left\|\mathbf{u}-\mathbf{v}^{h}\right\|_{0, \Omega}+\left\|\mathbf{u}^{h}-\mathbf{v}^{h}\right\|_{0, \Omega},
$$

the desired result (4.1) follows with $C=1+C_{3}$.

We now make the following assumptions:

(i) There is an $s \geq 0$ such that $\mathbf{u} \in V \cap\left(H^{s}(\Omega)\right)^{2}$.

(ii) $\left\{V^{h}\right\}_{h>0}$ is a regular family of finite elements, where $V^{h}$ is a subspace of $V$ consisting of piecewise polynomials of degree $k$, where $k \leq s-1$ (and thus, $\mathbf{u} \in\left(H^{k+1}(\Omega)\right)^{2}$ ).

Then we have the following error estimate.

Theorem 4.2. Suppose that the hypotheses of Theorem 4.1, and (i), (ii) hold. Then there is a constant $C>0$ depending only on $k_{1}$ and $k_{2}$ such that

$$
\left\|\mathbf{u}-\mathbf{u}^{h}\right\|_{0, \Omega} \leq C h^{k}|\mathbf{u}|_{k+1, \Omega} .
$$

Proof. Immediate from Theorem 4.1 and the usual interpolation-theoretic results.

\section{EXAMPles}

We present here some examples to verify that the assumptions made in $\S 3$ are indeed not very restrictive. Some numerical implementations of the finite element method to a particular example will be given.

Example 1. Consider the following second-order parabolic equation:

$$
x \phi_{t}(x, t)-\phi_{x x}(x, t)=f(x, t) \quad \forall(x, t) \in \Omega,
$$

where $\Omega=(-1,1) \times(0,1)$, with the boundary conditions

$$
\begin{aligned}
\phi( \pm 1, t)=0 & \forall t \in[0,1], \\
\phi(x, 0)=0 & \forall x \in[0,1], \\
\phi(x, 1)=0 & \forall x \in[-1,0],
\end{aligned}
$$

where $f$ is chosen as

$$
f(x, t)=\left\{\begin{array}{l}
2 x\left(x^{2}-1\right) t\left[(t-1)^{2}-4 x^{2}+t(t-1)\right] \\
-2 t^{2}\left[(t-1)^{2}-24 x^{2}+4\right] \quad \forall x \geq 0, t \in[0,1], \\
2 x\left(x^{2}-1\right)(t-1)\left(2 t^{2}-t-4 x^{2}\right) \\
-2(t-1)^{2}\left(t^{2}-24 x^{2}+4\right) \quad \forall x \leq 0, t \in[0,1] .
\end{array}\right.
$$

It is easy to show that

$$
\phi(x, t)= \begin{cases}\left(x^{2}-1\right) t^{2}\left[(t-1)^{2}-4 x^{2}\right] & \forall x \geq 0, t \in[0,1] \\ \left(x^{2}-1\right)\left(t^{2}-4 x^{2}\right)(t-1)^{2} & \forall x \leq 0, t \in[0,1]\end{cases}
$$


is an exact solution to the boundary value problem (5.1), (5.2). This typical example will be used for all numerical calculations.

Example 2. For $\sigma(x, t)=x^{m}$ with $m$ an odd positive integer, we choose $\lambda=0.1, \alpha=1$, and $\beta=x^{-m+1}$. We then have

$$
\begin{aligned}
& H 1: \frac{1}{2}\left(1+0.2 x^{m}\right) \geq 0.4=k_{1}, \\
& H 2: 1=k_{2}, \\
& H 3: x<2 \sqrt{0.4}=1.2649, \\
& H 4:\left.x^{m} n_{t}\right|_{\Gamma_{1} \cup \Gamma_{4}} \geq 0 .
\end{aligned}
$$

Example 3. We now give an example for which $\sigma(x, t)=x+\frac{1}{8} t$. Let $\lambda=0.1$, $\alpha=2$, and $\beta=1$. We then have

$$
\begin{aligned}
& H 1: \frac{3}{8}+0.2\left(x+\frac{1}{8} t\right) \geq \frac{7}{40}=k_{1}, \\
& H 2: 2=k_{2}, \\
& H 3: x+\frac{1}{8} t \leq \frac{9}{8}=1.125<2 \sqrt{\frac{14}{40}}=1.1832, \\
& H 4:\left.\left(x+\frac{1}{8} t\right) n_{t}\right|_{\Gamma_{1} \cup \Gamma_{4}} \geq 0 .
\end{aligned}
$$

For the finite element procedure, we formulate (5.1) as a first-order system which is not symmetric positive.

Now, the parameters $\lambda, \alpha$, and $\beta$ are chosen as

$$
\lambda=0.1, \quad \alpha=2, \quad \beta=2 .
$$

If

$$
\mathbf{u}=\left[\begin{array}{l}
u_{1} \\
u_{2}
\end{array}\right]=\left[\begin{array}{c}
e^{-0.1 t} \phi \\
e^{-0.1 t} \phi_{x}
\end{array}\right],
$$

then using (5.1), we obtain the system of first-order equations

$$
\begin{aligned}
L \mathbf{u} & =A_{1} \mathbf{u}_{x}+A_{2} \mathbf{u}_{t}+A_{3} \mathbf{u} \\
& =\left[\begin{array}{cc}
0 & -1 \\
-1 & 0
\end{array}\right] \mathbf{u}_{x}+\left[\begin{array}{ll}
x & 0 \\
0 & 0
\end{array}\right] \mathbf{u}_{t}+\left[\begin{array}{cc}
-0.1 x & 0 \\
0 & 1
\end{array}\right] \mathbf{u} \\
& =\left[\begin{array}{c}
e^{-0.1 t} f \\
0
\end{array}\right] \text { in } \Omega,
\end{aligned}
$$

with boundary condition

$$
u_{1}(x, t)=0 \quad \forall(x, t) \in \Gamma_{2} \cup \Gamma_{3} \cup \Gamma_{5} \cup \Gamma_{6} .
$$

With the choice of (5.3), we then have the bounded operator

$$
T=\left[\begin{array}{cc}
2 & 0 \\
2 x & 2
\end{array}\right]
$$


Let us verify the hypotheses

$$
\begin{aligned}
& H 1: 1+0.2 x \geq \frac{4}{5}=k_{1}, \\
& H 2: 2=k_{2}, \\
& H 3: 2 x<2 \sqrt{\frac{8}{5}}, \\
& H 4:\left.x n_{t}\right|_{\Gamma_{1} \cup \Gamma_{4}} \geq 0 .
\end{aligned}
$$

After subdividing $\Omega$ into squares, we choose the space of approximating functions $V^{h}$ as the set of piecewise bivariate polynomials with degree $\leq 2$ on the squares which satisfy boundary condition (5.6).

In Table 5.1 we see the $L^{2}$ error and the $L^{2}$ rate of convergence for various mesh sizes $h$. These results show $\mathbf{O}\left(h^{2}\right)$ accuracy.

\section{TABLE 5.1}

Finite element computation

\begin{tabular}{|c|c|c|c|}
\hline$h$ & $\max |\mathbf{e}|$ & $L^{2}$ error & $L^{2}$ rate \\
\hline$\frac{1}{2}$ & 4.271 & 1.104 & \multirow{2}{*}{1.99} \\
\hline$\frac{1}{4}$ & 1.208 & 0.276 & 2.02 \\
\cline { 1 - 2 }$\frac{1}{8}$ & 0.316 & 0.067 & 2.01 \\
$\frac{1}{16}$ & 0.078 & 0.016 & \\
\hline
\end{tabular}

\section{BIBLIOGRAPHY}

1. A. K. Aziz and P. Monk, Continuous finite elements in space and time for the heat equation, Math. Comp. 52 (1989), 255-274.

2. A. K. Aziz, S. Leventhal, and A. Werschulz, Higher-order convergence for a finite element method for the Tricomi problem, Numer. Funct. Anal. Optim. 2 (1980), 65-78.

3. M. S. Baouendi and P. Grisvard, Sur une équation d'évolution changeant de type, J. Funct. Anal. 2 (1968), 352-367.

4. K. O. Friedrichs, Symmetric positive differential equations, Comm. Pure Appl. Math. 11 (1958), 333-418.

5. M. Gevrey, Sur les équations aux dérivées partielles du type parabolique, J. Math. Pures Appl. (6) 9 (1913), 305-475.

6. __ Sur les équations aux dérivées partielles du type parabolique (suite), J. Math. Pures Appl. (6) 10 (1914), 105-148.

7. J. A. Goldstein and T. Mazumdar, A heat equation in which the diffusion coefficient changes sign, J. Math. Anal. Appl. 103 (1984), 533-564.

8. T. LaRosa, The propagation of an electron beam through the solar corona, Ph.D. Dissertation, Department of Physics and Astronomy, University of Maryland, 1986.

9. P. Lesaint and P. A. Raviart, Finite element collocation methods for first order systems, Math. Comp. 33 (1979), 891-918.

10. J. Lions, Quelques méthodes de résolution des problèmes aux limites non linéaires, Dunod Gauthier-Villars, Paris, 1969. 
11. K. Stewartson, Multistructural boundary layers on flat plates and related bodies, Adv. in Appl. Mech. 14 (1974), 145-239.

12. __ D'Alembert's paradox, SIAM Rev. 23 (1981), 308-343.

13. V. Thomée, Galerkin finite element methods for parabolic problems, Lecture Notes in Math., vol. 1054, Springer-Verlag, 1972.

14. V. Vanaja, Iterative solutions of backward-forward heat equation, Ph.D. Dissertation, Department of Mathematics, University of Maryland, 1988.

Department of Mathematics, University of Maryland Baltimore County, Baltimore, MARYLAND 21228

Department of Mathematics and Statistics, University of Pittsburgh, Pittsburgh, PennsylVania 15260 